\title{
Star Formation Induced by Cloud-Cloud Collisions and Galactic Giant Molecular Cloud Evolution
}

\author{
Masato I.N. KOBAYASHI ${ }^{1}$, Hiroshi KOBAYASHI ${ }^{1}$, Shu-ichiro INUTSUKA ${ }^{1}$, \\ Yasuo FUKUI ${ }^{1}$ \\ ${ }^{1}$ Division of Particle and Astrophysical Science, Graduate School of Science, Nagoya \\ University, Aichi 464-8602, Japan \\ *E-mail: masato.kobayashi@nagoya-u.jp \\ Received 〈reception date〉; Accepted 〈acception date〉
}

\begin{abstract}
Recent radio observations towards nearby galaxies started to map the whole disk and to identify giant molecular clouds (GMCs) even in the regions between galactic spiral structures. Observed variations of GMC mass functions in different galactic environment indicates that massive GMCs preferentially reside along galactic spiral structures whereas inter-arm regions have many small GMCs. Based on the phase transition dynamics from magnetized warm neutral medium to molecular clouds, Kobayashi et al. 2017 proposes a semi-analytical evolutionary description for GMC mass functions including cloud-cloud collision (CCC) process. Their results show that CCC is less dominant in shaping the mass function of GMCs compared with the accretion of dense $\mathrm{HI}$ gas driven by the propagation of supersonic shock waves. However, their formulation does not take into account the possible enhancement of star formation by CCC. Radio observations within the Milky Way indicate the importance of CCC for the formation of star clusters and massive stars. In this article, we reformulate the time evolution equation largely modified from Kobayashi et al. 2017 so that we additionally compute star formation subsequently taking place in CCC clouds. Our results suggest that, although CCC events between smaller clouds are more frequent than the ones between massive GMCs, CCC-driven star formation is mostly driven by massive GMCs $\gtrsim 10^{5.5} M_{\odot}$ (where $M_{\odot}$ is the solar mass). The resultant cumulative CCC-driven star formation may amount to a few 10 per cent of the total star formation in the Milky Way and nearby galaxies.
\end{abstract}

Key words: ISM: clouds — ISM: bubbles — Galaxies: star formation — Galaxies: evolution

\section{INTRODUCTION}

Giant molecular clouds (GMCs) are believed to be the parental structure of hydrogen molecules $\left(\mathrm{H}_{2}\right)$ forming stars, which have a typical mass $\gtrsim 10^{4} M_{\odot}$, where $M_{\odot}$ is the solar mass, and $\gtrsim 10$ parsec (pc) (e.g., Williams et al. 2000; Kennicutt $\&$ Evans 2012). Understanding of star formation and subsequent galaxy evolution therefore requires a framework to describe GMC formation, evolution, and dispersal on galactic scales. Over the last 10 years, radio observations towards nearby galaxies have started to map GMC distributions throughout their entire disks (Engargiola et al. 2003; Rosolowsky et al. 2003, 2007; Koda et al. 2009, 2011, 2012; Colombo et al. 2014a, 2014b). Especially, the Plateau de Bure Interferometer (PdBI) Arcsecond Whirlpool Survey (PAWS) program observed Galaxy M51 in detail with PdBI and the IRAM 30m telescope (Schinnerer et al. 2013). One of the highlighted results is the observed variation in GMC mass function (GMCMF) (Colombo et al. 2014a); when fitted with a power-law profile

(C) 2014. Astronomical Society of Japan. 
$n_{\mathrm{cl}}(m) \propto m^{-\alpha}$, where $n_{\mathrm{cl}}(m)$ represents the differential number density of GMC with mass $m$ (i.e., the cumulative number density of GMCs with mass greater than $m, n(>m)$, is given as $\left.n(>m)=\int_{m}^{\infty} n_{\mathrm{cl}}(m) \mathrm{d} m\right)$, the GMCMF shows a shallower slope $-\alpha \sim-1.3$ in arm regions whereas $-\alpha \sim-2.6$ in interarm regions. This result indicates that massive GMCs preferentially reside along galactic spiral arms whereas the mass budget in inter-arm regions is dominated by less massive GMCs $\left(m<10^{5.5} M_{\odot}\right)$. It is therefore required to connect such observed trends in ensemble GMC populations with the phase transition dynamics on pc and sub-pc scales (as mentioned in the following paragraph) for understanding GMC evolution as well as subsequent star formation and galaxy evolution.

The interstellar medium (ISM) constructs thermally bistable phases of atomic hydrogen due to the balance between radiative cooling and photoelectric heating (partially cosmic ray heating as well) (Field et al. 1969; Wolfire et al. 1995, 2003). One of the two phases is warm neutral medium (WNM) with the temperature $\sim 6000 \mathrm{~K}$ and the density $\sim 0.1$ atomic hydrogen $\mathrm{cm}^{-3}$, and the other phase is cold neutral medium (CNM) with the temperature $\sim 100 \mathrm{~K}$ and the density $\sim 100$ atomic hydrogen $\mathrm{cm}^{-3}$, which is a precursor of GMCs. WNM occupies most of the volume in galactic disks, and thus the phase transition dynamics from WNM to CNM is important for GMC formation on galactic scales. Over the last 20 years, multiphase ISM simulations investigate the propagation of shock waves in WNM to override the pressure balance between WNM and CNM, and they successfully form molecular clouds from WNM through thermal instability (e.g. Walder \& Folini 1998a, 1998b; Koyama \& Inutsuka 2002; Audit \& Hennebelle 2005, 2008; Heitsch et al. 2005, 2006; Vázquez-Semadeni et al. 2006; Hennebelle \& Audit 2007; Hennebelle et al. 2007). Here, shock waves are supposed to be driven by expanding supernovae or HII regions in the real ISM. However, multiphase magnetohydrodynamics ISM simulations since about 10 years ago have revealed that magnetic field pressure can support the ISM against compression driven by shock waves, so that magnetic fields retard the cloud formation from magnetized WNM with a typical field strength of just a few micro Gauss in the ISM (e.g. Inoue \& Inutsuka 2008). These results suggest that successful molecular cloud formation takes place only after a few 10 multiple shocks compress WNM with the shock propagation directions misaligned with local magnetic field lines. Therefore, multiple episodes of supersonic compression is presumably essential to form molecular clouds from magnetized WNM, and the typical phase transition timescale by such multiple compression is estimated about a few 10 Myr (Inutsuka et al. 2015).

To connect individual GMC formation governed by multiple episodes of compression with the evolution of GMC populations over galactic disks, Inutsuka et al. (2015) propose a bubble scenario where the network of expanding shells due to expanding supernovae and HII regions create repeated supersonic shock propagations. Based on this paradigm, they formulate a time evolution equation of GMCMF due to the multiple episodes of compression and GMC self-dispersal. However, this formulation neglected the change of cloud mass function by cloud-cloud collisions (CCCs). From 1970s, GMC evolution due to CCCs alone is extensively investigated by coagulation equation (e.g., Kwan 1979; Scoville \& Hersh 1979; Cowie 1980) and N-body simulations (e.g., Levinson \& Roberts 1981; Kwan \& Valdes 1983; Tomisaka 1984, 1986). Thus, in the previous studies of the cloud mass function, the detailed formation/dispersal of clouds and CCC were studied separately. These studies are separately conducted but have to be incorporated to reveal which process plays a dominant role in shaping what part of GMCMF evolution.

Kobayashi et al. (2017) extended the formulation in Inutsuka et al. (2015) by including CCCs. Their results indicate that CCC modifies only the massive end of GMCMF while GMCMF exhibits a power-law slope in the low mass regime $\left(\lesssim 10^{5.5} M_{\odot}\right)$, which is well characterized by a combination of two timescales: formation/growth and dispersal (see section 2 in this article). CCC determines the power-law slope of the cloud mass function only when these formation and dispersal are slower processes than CCC (e.g., such as galactic centers where GMC number densities are higher than disk regions; c.f., Kwan 1979; Cowie 1980; Tomisaka 1984). However, Kobayashi et al. (2017) focus only on gas phase, namely GMCMF, so that they have not investigated resultant star formation out of those GMC populations. Indeed, recent radio observations have found increasing number of star cluster forming sites likely triggered by CCC (e.g., Torii et al. 2011; Nakamura et al. 2012; Fukui et al. 2014; Torii et al. 2015; Fukui et al. 2016; Torii et al. 2017c, 2017b; Nishimura et al. 2017b, 2017a; Fukui et al. 2017c, 2017a, 2017e, 2017d; Sano et al. 2017b; Ohama et al. 2017a, 2017b; Kohno et al. 2017; Hayashi et al. 2017; Saigo et al. 2017; Tsutsumi et al. 2017; see also Furukawa et al. 2009; Ohama et al. 2010; Dobashi et al. 2014; Nakamura et al. 2014; Fukui et al. 2015; Tsuboi et al. 2015; Dewangan et al. 2016; Dewangan 2017; Ohama et al. 2017c; Sano et al. 2017a; Torii et al. 2017a) Their interpretation of CCC indicates the possible importance of CCC-driven star formation across the Milky Way galaxy from the solar circle to the Galactic Center. Simulations of colliding GMCs (e.g., Inoue \& Fukui 2013; Takahira et al. 2014; Inoue et al. 2017; Wu et al. 2017; Takahira et al. 2017) also suggest molecular cloud core formation in a shocked compressed layer, which may result in rapid star cluster formation and efficient massive star formation.

In this article, we introduce star formation rate (SFR) implementation to our time-evolution equation for GMCMF from Kobayashi et al. (2017), and calculate SFR with a given GMCMF to evaluate the relative contribution by $\mathrm{CCC}$ to to- 
tal star formation across galactic disks. We also evaluate CCC timescales as a function of GMC masses and discuss what mass pair is most likely to be observed.

This article is organized as follows. In section 2, we briefly review the time-evolution equation on GMCMF formulated in Kobayashi et al. (2017). In section 3, we introduce our new time-evolution equation revised from Kobayashi et al. (2017) and introduce equations that calculate SFR. In section 4, we explore our results: GMCMF with triggered star formation in CCC sites, CCC-driven SFR, and CCC frequency as a function of GMC masses. The possible improvements in our calculations are listed in section 5, and section 6 summarizes this article. All the "Log" appeared in the figure labels are logarithm in the base of 10 .

\section{Basic Evolution Equation for Giant Molecular Cloud Mass Function}

Our formulation in this study is extensively based on our time evolution of GMCMF from equation (1) in Kobayashi et al. (2017). Thus in this section, we briefly summarize Kobayashi et al. (2017) and refer the readers to Kobayashi et al. (2017) for the detail assumptions and formulations if necessary.

The time evolution of the differential number density of GMCs with mass $m, n_{\mathrm{cl}}$, is evaluated as

$$
\begin{aligned}
\frac{\partial n_{\mathrm{cl}}}{\partial t} & +\frac{\partial}{\partial m}\left(n_{\mathrm{cl}}\left(\frac{\mathrm{d} m}{\mathrm{~d} t}\right)_{\mathrm{self}}\right) \\
& =-\frac{n_{\mathrm{cl}}}{T_{\mathrm{d}}} \\
& +\frac{1}{2} \int_{0}^{\infty} \int_{0}^{\infty} K\left(m_{1}, m_{2}\right) n_{\mathrm{cl}, 1} n_{\mathrm{cl}, 2} \\
& -\int_{0}^{\infty} K\left(m, m_{2}\right) n_{\mathrm{cl}} n_{\mathrm{cl}, 2} \mathrm{~d} m_{2}+\left.\frac{1}{m} \frac{\partial\left(n_{\mathrm{cl}} m\right)}{\partial t}\right|_{\text {res }} .
\end{aligned}
$$

Here, $(\mathrm{d} m / \mathrm{d} t)_{\text {self }}$ represents the mass-growth rate of GMCs due to accretion from the ambient ISM, $T_{\mathrm{d}}$ is the self-dispersal timescale of GMCs, $n_{\mathrm{cl}, 1}$ and $n_{\mathrm{cl}, 2}$ are the differential number density of GMCs whose masses are $m_{1}$ and $m_{2}$ respectively, $K\left(m_{1}, m_{2}\right)$ is the kernel function that determines the $\mathrm{CCC}$ rate between GMCs with mass $m_{1}$ and $m_{2}, \delta$ is the Dirac delta function, and $(1 / m) \partial\left(n_{\mathrm{cl}} m\right) /\left.\partial t\right|_{\text {res }}$ is the gas resurrection rate from dispersed gas. Throughout this article, we assume that GMCs are molecular agglomeration bright in ${ }^{12} \mathrm{CO}(1-0)$ line to be compared with observations.

We opt to employ $100 \mathrm{pc}$ as the disk scale height where GMCs populate, which is observed in the Milky Way galaxy (e.g., Dame et al. 1987). The scale height observationally indicated has a variation by a factor two to three (e.g., 35 pc (Stark $\&$ Lee 2005), half-luminosity height $\lesssim 60$ pc (Bronfman et al. 2000)), thus CCC rate may increase by at most a factor two to three because a smaller thickness of the galactic disk means a larger number density of molecular clouds in the disk.

\subsection{Self-Growth Term}

The second term on the left-hand side of equation (1) corresponds to a flux term in the conservation law. The continuity equation in fluid dynamics is one of such conservation laws, where mass is the conserved quantity in the configuration space. On the other hand, we here consider GMC number conservation in GMC mass space because the number should be the conserved quantity unless GMCs experience an abrupt change (e.g., dispersal or CCCs). This term, therefore, represents the GMC number flux in GMC mass space, which corresponds to GMC mass-growth in the configuration space. The mass-growth rate $(\mathrm{d} m / \mathrm{d} t)_{\text {self }}$ can be basically evaluated

$\left(\frac{\mathrm{d} m}{\mathrm{~d} t}\right)_{\text {self }}=\frac{m}{T_{\mathrm{f}}}$,

where $T_{\mathrm{f}}$ is the typical mass-growth timescale. This substitution is based on our assumption that the mass-growth rate (i.e., massgain rate from the ambient ISM) is proportional to GMC's surface area and the surface area is proportional to mass, if we employ the observational results that the majority of GMCs have a similar column density $2 \times 10^{22} \mathrm{~cm}^{-2}$ (Onishi et al. 1999; Tachihara et al. 2000). The typical mass-growth timescale, $T_{\mathrm{f}}$, can have the same order of magnitude with the typical phase transition timescale if the mass-growth of molecular clouds are driven by the phase transition from the ambient WNM to molecular gas on the surface of GMCs, similar to the molecular cloud formation. Such phase transition timescale is evaluated about a few $10 \mathrm{Myr}$, over which molecular clouds successfully form out of magnetized WNM by repeated supersonic shocks from random direction due to expanding bubbles (see Inutsuka et al. (2015); Kobayashi et al. (2017)). Therefore, we opt to choose $T_{\mathrm{f}}=10 \mathrm{Myr}$ as our fiducial timescale. Our bubble paradigm is less likely to create large GMCs whose mass is comparable with or exceeds the total gas mass that a single supernova can sweep, thus we model such a cut-off mass scale $\sim 7 \times 10^{6} M_{\odot}$ beyond which $T_{\mathrm{f}}$ becomes virtually infinite (see equation (4) in Kobayashi et al. (2017)).

Note that, in principle, $T_{\mathrm{f}}$ is the ensemble averaged timescale over different GMC mass-growth processes, for which we here consider the multiple episodes of supersonic compressions is the most important under the magnetic fields. The relative importance of different mass-growth processes depending on galactic environments needs to be further investigated in the future.

\subsection{Dispersal Term}

The first term on the right-hand side of equation (1) means the GMC self-dispersal due to stellar feedback from massive stars born within those GMCs. Here, this feedback can be any means (ionization, dissociation, heating, blowing-out, etc.). The characteristic dispersal timescale, $T_{\mathrm{d}}$, can be evaluated as 
$T_{\mathrm{d}}=T_{*}+T_{\text {dest }}$,

where $T_{*}$ is the typical timescale for the protostars to evolve into the main-sequence stars after the birth of the concerned GMC, and $T_{\text {dest }}$ is the typical timescale for the complete destruction of GMCs after the star formation onset. According to recent theories and observations within the Milky Way galaxy, the filamentary structure in densest parts of GMCs may host most of star formation in GMCs (e.g., Inutsuka 2001; André et al. 2010, 2011; Roy et al. 2015), and such filaments too can form through multiple supersonic shocks (c.f., Inoue \& Inutsuka 2012; Inutsuka et al. 2015). Therefore, we assume that $T_{*}$ would have a similar timescale as $T_{\mathrm{f}}$ so that we employ $T_{*} \sim 10$ Myr. According to line-radiation magnetohydrodynamics simulations (e.g., results in Inutsuka et al. (2015) which updates Hosokawa \& Inutsuka (2006) by including magnetic fields), the typical timescale for the dissociation of $\mathrm{CO}$ molecules is estimated as $T_{\text {dest }} \sim 4 \mathrm{Myr}$ and is irrespective of parental GMC mass (see section 4.2 for the justification of this mass-independency argument). Therefore, we expect that the typical dispersal timescale $T_{\mathrm{d}}=10+4=14 \mathrm{Myr}$. Due to its definition, $T_{\mathrm{d}}$ essentially measures the typical time-scale over which GMCs are no longer identified in CO line observations. Thus this formulation implicitly allows the formation of COdark molecular gas (hereafter CO-dark gas) whose population is left to be studied in the future.

Note that $T_{\mathrm{d}}$ does not always guarantee complete blowout of GMCs physically. Meanwhile, several detailed semianalytical studies (e.g., Kim et al. 2016; Rahner et al. 2017) report that, in some range of initial conditions of hydrogen number density in GMCs, GMC mass, and star cluster mass, stellar feedback from a single star cluster (both wind and radiation) cannot completely blow out GMCs because of the gravity between the swept-up shell and the star cluster. This complete blow-out process as well as CO-dark gas population also need to be investigated in the future.

Note that, similarly to $T_{\mathrm{f}}, T_{\mathrm{d}}$ is the ensemble averaged timescale over different GMC destructive processes. Therefore, other processes may play an important role as well in different galactic environments; for example, galactic shear may dominate in much inner regions in galactic disks (c.f. Dobbs \& Pringle 2013). The relative importance of different destructive processes needs to be further studied in the future (c.f., Jeffreson 2017 in prep.)

\subsection{Cloud-Cloud Collision Terms}

The second and third integration terms on the right-hand side of equation (1) represent CCC, whose formulation is essentially the same as the coagulation of two colliding dust particles in protoplanetary disks (e.g., Trubnikov 1971; Malyshkin \& Goodman 2001). The first term in the two calculates the formation of GMCs with mass $m$ through the CCC between GMCs with mass $m_{1}$ and $m_{2}$. The second term in the two calculates the formation of GMCs with mass $m+m_{2}$ through the CCC between GMCs with mass $m$ and $m_{2}$. Therefore, to highlight the CCC effect simply, our formulation considers $\mathrm{CCC}$ as a coagulation process. The CCC kernel function $K\left(m_{1}, m_{2}\right)$ is the product of the total collisional cross section between GMCs with mass $m_{1}$ and $m_{2}, \sigma_{\mathrm{col} 1,2}$, and the relative velocity between the GMCs, $V_{\text {rel }}$,

$K\left(m_{1}, m_{2}\right)=\sigma_{\mathrm{col} 1,2} V_{\mathrm{rel}}=c_{\mathrm{col}} \frac{m_{1}+m_{2}}{\Sigma_{\mathrm{mol}}} V_{\mathrm{rel}, 0}$.

Here, $c_{\mathrm{col}}$ is a correction factor, $\Sigma_{\mathrm{mol}}$ is a typical column density of GMCs, and $V_{\mathrm{rel}, 0}$ is a typical relative velocity between GMCs. Note that, in equations (1) and (4), we restrict ourselves only to a perfect inelastic collision case (i.e., coagulation) for simplicity.

The total collisional cross section can be essentially evaluated as the total geometrical cross section of two colliding GMCs. The GMC geometrical surface area can be estimated as their mass divided by a characteristic column density $m / \Sigma_{\text {mol }}$, given the observational fact that the majority of GMCs have a constant column density of a few times $10^{22} \mathrm{~cm}^{-2}$ if averaged over the entire cloud scale (e.g., Onishi et al. 1999; Tachihara et al. 2000; see also subsection 5.1 for its variation). We opt to employ an observed value $\Sigma_{\mathrm{mol}}=2 \times 10^{22} \mu m_{\mathrm{H}} \mathrm{cm}^{-2}$, where $\mu$ is the mean molecular weight and $m_{\mathrm{H}}$ is the atomic hydrogen weight. Observationally, the cloud-to-cloud velocity dispersion is measured as $8-10 \mathrm{~km} \mathrm{~s}^{-1}$ (Stark \& Brand 1989; Stark \& Lee 2005, 2006). The bubble paradigm predicts that GMCs are repeatedly pushed by supersonic shocks due to expanding shells and thus the sound speed of the medium within those expanding shells set the GMC velocity dispersion, which is about $10 \mathrm{~km}$ $\mathrm{s}^{-1}$. Therefore, observed velocity dispersion is consistent with our bubble paradigm and we opt to set $V_{\mathrm{rel}, 0}=10 \mathrm{~km} \mathrm{~s}^{-1}$. Note that we turn off CCC calculations that involve GMCs whose cumulative number is less than 1, because such GMC populations are less likely to exist in the real Universe (see also section 4 in Kobayashi et al. (2017) for the detail and also subsection 5.1 of this article).

Several variations (e.g., gravitational focusing effect, angle variation at which GMCs collide with each other) may make the total collisional cross section differ from the total geometrical cross section. A factor of few differences due to these variations may impact the GMCMF massive-end evolution and the computed total SFR on the entire galactic disks, but do not on the power-law slope (see Kobayashi et al. (2017)). Thus, for simplicity, we opt to choose $c_{\mathrm{col}}=1$ (see also subsection 5.1 for other details involved in $\left.c_{\mathrm{col}}\right)$. 


\subsection{Gas Resurrection}

The dispersal term in equation (1) produces dispersed gas. However, this term alone does not restore dispersed gas back into GMC populations. In reality when GMCs disperse, they turn into ambient ISM in several phases: ionized, atomic, COdark, optically thick Hi etc. Irrespective of phases, those dispersed gas may experience repeated supersonic shocks while floating around in the ISM to form a newer generation of GMCs or to accrete onto pre-existing GMCs to help their mass-growth. Hereafter, we call this process as "gas resurrection" following the nomenclature named in Kobayashi et al. (2017). The last term in equation (1) represents this gas resurrection. To calculate gas resurrection, we introduce "gas resurrecting factor", $\varepsilon_{\text {res }}$, in Kobayashi et al. (2017), which is the mass fraction that is consumed to form newer generation of the minimum-mass GMCs out of the total amount of dispersed gas:

$$
\left.\frac{\partial\left(n_{\mathrm{cl}} m\right)}{\partial t}\right|_{\text {res }}=\varepsilon_{\text {res }} \dot{\rho}_{\text {total }, \text { disp }} \delta\left(m-m_{\text {min }}\right),
$$

Here, $\dot{\rho}_{\text {total, disp }}$ is the total amount of dispersed gas produced from the system per unit time per unit volume, and $m_{\min }$ is the minimum GMC mass (i.e., $10^{4} M_{\odot}$ in this article). By this definition, in the steady state case, $1-\varepsilon_{\text {res }}$ fraction of the dispersed gas is consumed to help the mass-growth of pre-existing intermediate-mass GMCs, whose rate is given by the flux term $m / T_{\mathrm{f}}$ in equation (1).

\subsection{Steady State Solution}

Kobayashi et al. (2017) reveal that the CCC impact is limited only in the massive-end evolution of GMCMF. Therefore, the power-law GMCMF feature in lower mass regime can be characterized by a steady state solution of the time-evolution equation without the CCC terms as

$n_{\mathrm{cl}}(m)=n_{0}\left(\frac{m}{M_{\odot}}\right)^{-1-\frac{T_{\mathrm{f}}}{T_{\mathrm{d}}}}$.

Here, $n_{0}$ is the differential number density normalized at $m=$ $M_{\odot}$. This solution indicates that ongoing and future large radio surveys with higher spatial resolution and higher sensitivity may constrain the timescale ratio $T_{\mathrm{f}} / T_{\mathrm{d}}$ by identifying smaller GMCs and measuring the power-law slope in $\mathrm{GMCMF}^{1}$.

Equation (6) indicates that $T_{\mathrm{f}}$ can vary from 4 to $22 \mathrm{Myr}$ to reproduce observed variation in GMCMF slope given that $T_{\mathrm{d}}$ is presumably determined more by stellar evolution but not by galactic environment (e.g., arm or inter-arm; see Kobayashi et al. (2017)). Indeed for example, based on PAWS data on

\footnotetext{
${ }^{1}$ In a crowded region such as galactic centers, the number density of GMCs is higher than in disk regions. CCC may be a faster process than massgrowth or self-dispersal. In such cases, the power-law slope varies with the dependence of the kernel function $K$ on GMC masses. See for this analysis in, for example, equation (A4) in Kwan (1979) and equation (31) in Kobayashi et al. (2017).
}

Galaxy M51, Leroy et al. (2017) report that the depletion timescale due to star formation is almost constant with the total molecular column density in $\mathrm{CO}(1-0)$ line averaged on $40 \mathrm{pc}$ scale where the depletion timescale is defined as the total amount of molecular gas divided by SFR. Their derived depletion timescale $\sim 2$ Gyr and star formation efficiency $\sim 0.3$ per cent gives $\sim 6 \mathrm{Myr}$ as individual GMC dispersal timescale, which is a factor shorter than our fiducial dispersal timescale $T_{\mathrm{d}}=14$ Myr. This factor difference $(14 / 6=2.3)$ may arise from shorter $T_{\mathrm{d}}$ in GMCs undergoing CCC (see subsection 3.2) but needs to be further investigated.

\section{REFORMULATION INCLUDING STAR FORMATION INDUCED BY CLOUD-CLOUD COLLISIONS}

In our previous time evolution equation introduced in equation (1), we do not implement any rapid star formation triggered by CCC. However, observations of compact star cluster forming sites (e.g. Torii et al. 2011; Kudryavtseva et al. 2012; Torii et al. 2015; Fukui et al. 2016, 2017e; Kohno et al. 2017) indicate that GMCs are likely to form stars effectively (within a short timescale $\lesssim 1 \mathrm{Myr}$ ) after GMCs experience CCC, because of drastic compression of WNM and high accretion rate by enhanced sound velocity (c.f., Inoue et al. 2017). Increasing number of CCC-candidate clouds reported from radio observations (e.g. Fukui et al. 2014, 2016) and the indication of frequent CCC events in galactic disk simulations (e.g., Tasker \& Tan 2009; Dobbs et al. 2015) suggest the importance in the investigation of the impact of CCC-driven star formation onto GMCMF evolution and its relative contribution to SFR for the entire galactic disks.

Before calculating the SFR, we first introduce a revised version of time evolution equation for GMCMF, by specifying the evolution of GMCs that are undergoing the feedback from CCC-driven star cluster formation. To do this, we subdivide GMC populations into two: the differential number density of GMCs of mass $m$ without experiencing CCC, $n_{\text {acc,cl }}(m)$, and the one with CCC experience, $n_{\mathrm{col}, \mathrm{cl}}(m)$. Hereafter, we call the GMC populations in $n_{\mathrm{acc}, \mathrm{cl}}(m)$ as "normal" GMCs and the ones in $n_{\mathrm{col}, \mathrm{cl}}(m)$ as "CCC" GMCs. The total differential number density of GMCs with mass $m, n_{\mathrm{cl}}(m)$ is given as

$n_{\mathrm{cl}}(m)=n_{\mathrm{acc}, \mathrm{cl}}(m)+n_{\mathrm{col}, \mathrm{cl}}(m)$.

The basic evolution follows the same equation as equation (1), but only CCC GMCs would have a shorter timescale for $T_{\mathrm{d}}$. Thus the revised evolution equation becomes

$$
\begin{aligned}
& \frac{\partial\left(n_{\mathrm{acc}, \mathrm{cl}}+n_{\mathrm{col}, \mathrm{cl}}\right)}{\partial t}+\frac{\partial}{\partial m}\left(\left(n_{\mathrm{acc}, \mathrm{cl}}+n_{\mathrm{col}, \mathrm{cl}}\right) \frac{m}{T_{\mathrm{f}}}\right) \\
= & -\frac{n_{\mathrm{acc}, \mathrm{cl}}}{T_{\mathrm{d}}}-\frac{n_{\mathrm{col}, \mathrm{cl}}}{T_{\mathrm{d}, \mathrm{col}}}
\end{aligned}
$$




$$
\begin{aligned}
& +\frac{1}{2} \int_{0}^{\infty} \int_{0}^{\infty} K\left(m_{1}, m_{2}\right) \\
& \quad \times\left(n_{\mathrm{acc}, \mathrm{cl}, 1}+n_{\mathrm{col}, \mathrm{cl}, 1}\right)\left(n_{\mathrm{acc}, \mathrm{cl}, 2}+n_{\mathrm{col}, \mathrm{cl}, 2}\right) \\
& \quad \times \delta\left(m-m_{1}-m_{2}\right) \mathrm{d} m_{1} \mathrm{~d} m_{2} \\
& -\int_{0}^{\infty} K\left(m, m_{2}\right) \\
& \quad \times\left(n_{\mathrm{acc}, \mathrm{cl}}+n_{\mathrm{col}, \mathrm{cl}}\right)\left(n_{\mathrm{acc}, \mathrm{cl}, 2}+n_{\mathrm{col}, \mathrm{cl}, 2}\right) \mathrm{d} m_{2} \\
& +\left.\frac{1}{m} \frac{\partial\left(n_{\mathrm{cl}} m\right)}{\partial t}\right|_{\mathrm{res}} .
\end{aligned}
$$

The subscripts 1 and 2 represent the mass bins $m_{1}$ and $m_{2}$ (e.g., $\left.n_{\text {acc }, \mathrm{cl}, 1}=n_{\text {acc }, \mathrm{cl}}\left(m_{1}\right)\right)$.

This equation can be separated into two equations in which we calculate the time-evolution of $n_{\text {acc,cl }}(m)$ and $n_{\text {col,cl }}(m)$ respectively. For normal GMCs,

$$
\begin{aligned}
& \frac{\partial n_{\mathrm{acc}, \mathrm{cl}}}{\partial t}+\frac{\partial}{\partial m}\left(m \frac{n_{\mathrm{acc}, \mathrm{cl}}}{T_{\mathrm{f}}}\right) \\
= & -\frac{n_{\mathrm{acc}, \mathrm{cl}}}{T_{\mathrm{d}}} \\
& -\frac{n_{\mathrm{acc}, \mathrm{cl}}}{n_{\mathrm{cl}}} \int_{0}^{\infty} K\left(m, m_{2}\right) \\
& \times\left(n_{\mathrm{acc}, \mathrm{cl}}+n_{\mathrm{col}, \mathrm{cl}}\right)\left(n_{\mathrm{acc}, \mathrm{cl}, 2}+n_{\mathrm{col}, \mathrm{cl}, 2}\right) \mathrm{d} m_{2} \\
& +\left.\frac{1}{m} \frac{\partial\left(n_{\mathrm{cl}} m\right)}{\partial t}\right|_{\mathrm{res}},
\end{aligned}
$$

and for CCC GMCs,

$$
\begin{aligned}
& \frac{\partial n_{\mathrm{col}, \mathrm{cl}}}{\partial t}+\frac{\partial}{\partial m}\left(m \frac{n_{\mathrm{col}, \mathrm{cl}}}{T_{\mathrm{f}}}\right) \\
&=- \frac{n_{\mathrm{col}, \mathrm{cl}}}{T_{\mathrm{d}, \mathrm{col}}} \\
&+\frac{1}{2} \int_{0}^{\infty} \int_{0}^{\infty} K\left(m_{1}, m_{2}\right) \\
& \quad \times\left(n_{\mathrm{acc}, \mathrm{cl}, 1}+n_{\mathrm{col}, \mathrm{cl}, 1}\right)\left(n_{\mathrm{acc}, \mathrm{cl}, 2}+n_{\mathrm{col}, \mathrm{cl}, 2}\right) \\
& \times \delta\left(m-m_{1}-m_{2}\right) \mathrm{d} m_{1} \mathrm{~d} m_{2} \\
&- \frac{n_{\mathrm{col}, \mathrm{cl}}}{n_{\mathrm{cl}}} \int_{0}^{\infty} K\left(m, m_{2}\right) \\
& \quad \times\left(n_{\mathrm{acc}, \mathrm{cl}}+n_{\mathrm{col}, \mathrm{cl}}\right)\left(n_{\mathrm{acc}, \mathrm{cl}, 2}+n_{\mathrm{col}, \mathrm{cl}, 2}\right) \mathrm{d} m_{2} .
\end{aligned}
$$

We give the ample descriptions on each term in the following subsections. All the parameters in this formulation are summarized in table 1.

\subsection{Self-Growth Term}

The second terms in equations (9) and (10) correspond to GMC mass-growth due to multiple episodes of supersonic compression. We assume that both normal population $n_{\text {acc,cl }}(m)$ and CCC population $n_{\mathrm{col}, \mathrm{cl}}(m)$ have the same $T_{\mathrm{f}}$ because the massgrowth driven by the phase transition dynamics presumably does not distinguish whether or not GMCs experience CCC. Therefore, the mass-growth rate for both populations can be characterized as $m / T_{\mathrm{f}}$ (see section 2.1 for the justification how $m / T_{\mathrm{f}}$ can be the mass-growth rate under the multiple episodes of supersonic compressions). A schematic flowchart of this mass-growth is shown as blue solid lines in figures 1 and 2 .

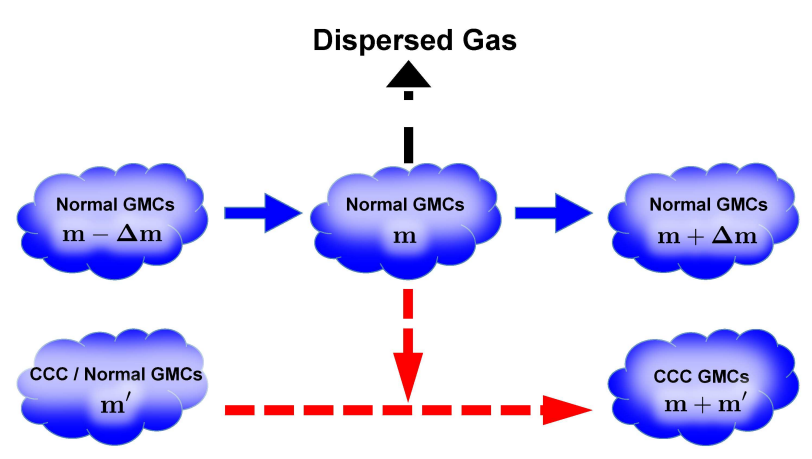

Fig. 1. Flowchart 1 describing the mass-growth, self-dispersal, and CCC of normal GMCs with mass $m$. The solid blue lines correspond to the mass-growth due to multiple episodes of supersonic compressions. Given a mass bin width $\Delta m$ in calculation, the multiple compressions grow GMCs from mass $m-\Delta m$ through $m$ to $m+\Delta m$. The red dashed lines show the CCC process. When normal GMCs with mass $m$ collide with GMCs with mass $m^{\prime}$ (either in normal or CCC populations), they coagulate together to create bigger GMCs with mass $m+m^{\prime}$, which join CCC populations but not normal populations. The black dot-dashed lines are GMC self-dispersal.

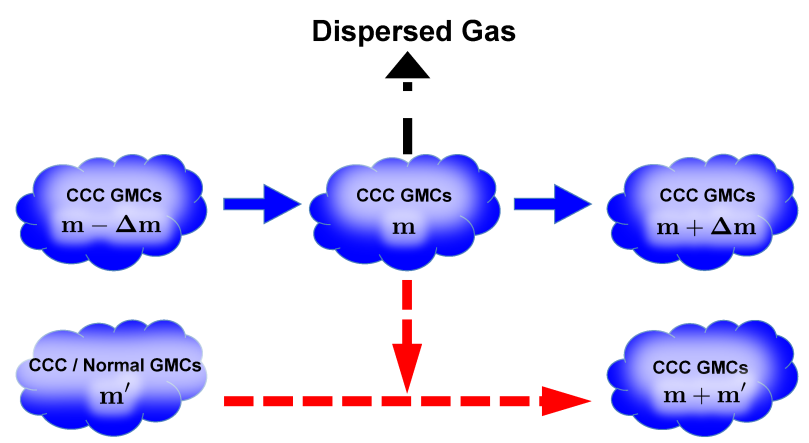

Fig. 2. Flowchart 2 describing the mass-growth, self-dispersal, and CCC of CCC GMCs with mass $m$. The solid blue lines correspond to the mass-growth due to multiple episodes of supersonic compressions. Given a mass bin width $\Delta m$ in calculation, the multiple compressions grow GMCs from mass $m-\Delta m$ through $m$ to $m+\Delta m$. Here, we assume that CCC GMCs remain in CCC populations through this mass-growth because they are undergoing stellar feedback triggered by CCC, which separates normal and CCC populations. The red dashed lines show the CCC process. When CCC GMCs with mass $m$ collide with GMCs with mass $m^{\prime}$ (either in normal or CCC populations), they coagulate together to create bigger GMCs with mass $m+m^{\prime}$, which join CCC populations but not normal populations. The black dot-dashed lines are GMC self-dispersal, which have a shorter characteristic dispersal timescale compared with the one in normal populations as discussed in subsection 3.2. 
Table 1. Parameters

\begin{tabular}{ccccc}
\hline \hline $\begin{array}{c}T_{\mathrm{f}} \\
(\mathrm{Myr})\end{array}$ & $\begin{array}{c}T_{\mathrm{d}} \\
(\mathrm{Myr})\end{array}$ & $\begin{array}{c}T_{\mathrm{d}, \mathrm{col}} \\
(\mathrm{Myr})\end{array}$ & $\varepsilon_{\text {res }}$ & $\varepsilon_{\mathrm{SFE}}$ \\
\hline 10 & 14 & 5 & 0.15 & 0.01 \\
\hline \hline
\end{tabular}

Note. Summary of the parameters in our formulation with their typical values. $T_{\mathrm{f}}$ denotes the mass-growth timescale of GMCs due to accretion of the ambient ISM by multiple episodes of compression. $T_{\mathrm{d}}$ is the GMC self-dispersal timescale due to the feedback by massive stars. $T_{\mathrm{d}, \mathrm{col}}$ is similar to $T_{\mathrm{d}}$ but is determined by massive stars that CCC events form. $\varepsilon_{\text {res }}$ denotes the resurrecting factor (i.e. the fractional mass out of total dispersed gas that replenishes the minimum-mass GMC population). $\varepsilon_{\mathrm{SFE}}$ represents the fractional mass converted into stars in CCC sites from their parental clouds. See also sections 2 and 3 for the detailed descriptions.

\subsection{Dispersal Term}

The first terms on the right hand side of equations (9) and (10) represent GMC self-dispersal due to stellar feedback by massive stars born within GMCs. Simulations of colliding GMCs (Inoue \& Fukui 2013; Takahira et al. 2014; Inoue et al. 2017) suggest triggering core formation in the shocked compressed layer. Especially, Inoue \& Fukui (2013) and Inoue et al. (2017) indicate that the effective sound speed and resultant effective Jeans mass increase in the layer so that CCC enables rapid massive star formation. In addition, observations suggest that GMCs undergoing CCC may form stars within a very short timescale $\lesssim 1$ Myr (c.f. Kudryavtseva et al. 2012; Fukui et al. 2016). We therefore assume that, with a shorter star formation timescale $T_{*}=1 \mathrm{Myr}$, CCC GMCs have their dispersal timescale $T_{\mathrm{d} \text {,col }}=T_{*}+T_{\text {dest }}=5$ Myr. A schematic flowchart of these dispersal processes is shown as black dot-dashed lines in figures 1 and 2.

From the observational viewpoint, the stellar initial mass function (IMF) might be a top-heavy in cluster forming regions (e.g., NGC6334: Muñoz et al. (2007), NGC3603:Harayama et al. (2008)). Magnetohydrodynamics simulations also demonstrate such top-heavy trend in CCC sites (at least before cores grow by mass accretion; e.g., Inoue \& Fukui (2013)). However for simplicity, we assume Salpeter IMF on the entire cloud scales even for GMCs undergoing or having undergone CCC. We opt to employ the same $T_{\text {dest }}=4 \mathrm{Myr}$ for both normal and CCC GMCs assuming that both GMC populations have the same dispersal efficiency with Salpeter IMF, whereas the star formation timescale $T_{*}$ alone is shorter for CCC populations.

\subsection{Cloud-Cloud Collision Terms}

The second term on the right hand side of equation (9) and the last two terms in equation (10) correspond to CCC process.
Equation (9) has only one term because CCC process decreases but never increase the normal GMC populations. Similarly to our previous study (Kobayashi et al. (2017) and section 2.3 in this article), we assume that $\mathrm{CCC}$ would work as a coagulation process so that colliding GMCs essentially form a larger GMC. Thus, the last term in equation (9) represents the formation of CCC GMCs with mass $m+m_{2}$ through the CCC between GMCs with mass $m$ and $m_{2}$. Similarly, the first CCC term in equation (10) represents the formation of CCC GMCs with mass $m$ through the CCC between GMCs with mass $m_{1}$ and $m_{2}$. Also, the second CCC term in equation (10) represents the formation of CCC GMCs with mass $m+m_{2}$ through the CCC between GMCs with mass $m$ and $m_{2}$. In this formulation, we assume a perfect inelastic collision for the CCC, as we did in our previous formulation shown in Equation (1).

We classify the resultant massive GMCs as CCC populations. This treatment restricts ourselves to assuming that rapid star formation is always invoked once GMCs experience CCC no matter what combination of GMC collide (i.e., collisions between normal populations, CCC populations, or normal and CCC populations). In this manner, GMCs become quickly dispersed once they experience CCC with a shorter dispersal timescale $T_{\mathrm{d} \text {,col }}$ compared with normal GMCs. A schematic flow of this CCC process is shown as red dashed lines in figures 1 and 2 .

Note that the CCC-driven star formation and subsequent stellar feedback in our calculation does not create any smaller GMCs and thus CCC GMCs simply disperse at a given rate of $1 / T_{\mathrm{d}, \mathrm{col}}$. The creation of such smaller GMCs by stellar feedback would impact the power-law slope in the low-mass regime, which needs to be investigated further in the future.

\subsection{Gas Resurrection}

The gas resurrection produces and replenishes the minimummass GMC populations. In this study, minimum-mass GMCs have only normal population but not $\mathrm{CCC}$ population because our CCC implementation does not produce any smaller mass clouds. Therefore, the gas resurrection term appears only in equation (9) but not in equation (10). This gas resurrection rate is calculated by equation (5). The mass production rate of dispersed gas, $\dot{\rho}_{\text {total,disp }}$, should be computed from both normal and CCC GMC populations thus is computed as

$\dot{\rho}_{\mathrm{total}, \mathrm{disp}}=\int \frac{m n_{\mathrm{acc}, \mathrm{cl}}}{T_{\mathrm{d}}} \mathrm{d} m+\int \frac{m n_{\mathrm{col}, \mathrm{cl}}}{T_{\mathrm{d}, \mathrm{col}}} \mathrm{d} m$.

Kobayashi et al. (2017) estimate that the steady state resurrecting factor for a typical galactic disk is about $\varepsilon_{\text {res }}=0.15$ (i.e., 15 per cent gas resurrection). We solve equation (8) simultaneously with equation (5) to calculate the gas resurrection. 


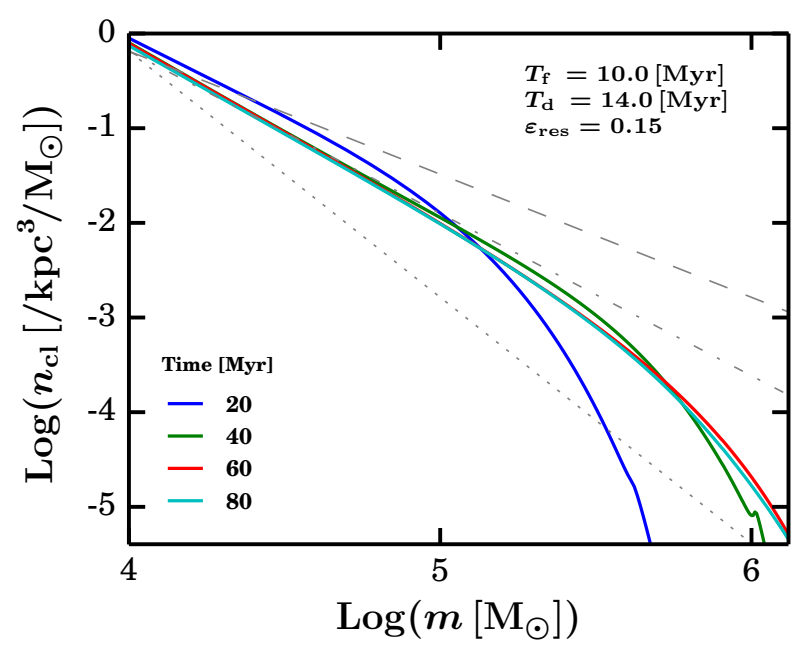

Fig. 3. Differential number density $n_{\mathrm{cl}}$ as a function of GMC mass, with $T_{\mathrm{f}}=10 \mathrm{Myr}, T_{\mathrm{d}}=14 \mathrm{Myr}, T_{\mathrm{d}, \mathrm{col}}=5 \mathrm{Myr}, \varepsilon_{\mathrm{res}}=0.15$. The color corresponds to time evolution. As a reference, we plot three thin gray lines; the dot-dashed line represents the steady state power-law slope $-1-T_{\mathrm{f}} / T_{\mathrm{d}} \sim-1.7$, the dashed line corresponds to the observed shallow slope in arm regions of Galaxy M51, and the dotted line corresponds to the observed steep slope in inter-arm regions of Galaxy M51. The calculated GMCMF shows a power-law slope $\lesssim 10^{5.5} M_{\odot}$ close to the steady state slope indicated by equation (6).

\section{RESULTS}

\subsection{Slope of Giant Molecular Cloud Mass Function}

We perform time integration of equation (8) coupled with equation (5). Figure 3 shows the resultant time evolution of GMCMF. We opt to employ our fiducial parameters (i.e., $\left.T_{\mathrm{f}}=10 \mathrm{Myr}, T_{\mathrm{d}}=14 \mathrm{Myr}, T_{\mathrm{d}, \mathrm{col}}=5 \mathrm{Myr}, \varepsilon_{\mathrm{res}}=0.15\right)$. The figure includes a reference dot-dashed line showing the steady state power-law slope characterized by equation (6). GMCMF in the mass range $m \lesssim 10^{5.5} M_{\odot}$ shows a single power-law slope close to this steady state solution. Compared with figure 7 in Kobayashi et al. (2017) where we calculated essentially the same condition but without CCC-driven star formation, the number of massive GMCs $\gtrsim 10^{6} M_{\odot}$ shown in figure 3 in this article decreases due to star formation driven by CCC and subsequent stellar feedback. Figure 3 also suggests that the powerlaw slope in the mass range $m \lesssim 10^{5,5} M_{\odot}$ is still preserved over the GMCMF evolution even with CCC-driven star formation. Therefore, our result indicates that CCC impacts only the massive-end of GMCMF.

\subsection{Star Formation Efficiency and Star Formation Rate}

To determine the relative contributions of normal and CCC GMCs onto star formation on galactic scales, we need to calculate SFR in each population. In this study, we opt to employ a given star formation efficiency (SFE) averaged over all GMC populations to calculate SFR coarse-grained on galactic scale.

Hereafter, we define SFE, $\varepsilon_{\mathrm{SFE}}$, as the final mass fraction that goes into stars from a parental GMC at the time when the entire GMC becomes completely dispersed. Cumulative SFR can be evaluated as the product of SFE and the dispersal term in equation (8):

$$
\begin{aligned}
\operatorname{SFR}(>m) & =\varepsilon_{\mathrm{SFE}} \\
& \times\left(\int_{m}^{\infty} \frac{m n_{\mathrm{acc}, \mathrm{cl}}}{T_{\mathrm{d}}} \mathrm{d} m+\int_{m}^{\infty} \frac{m n_{\mathrm{col}, \mathrm{cl}}}{T_{\mathrm{d}, \mathrm{col}}} \mathrm{d} m\right) .
\end{aligned}
$$

The first term corresponds to star formation due to normal GMC populations whereas the second term represents star formation originated in CCC GMC populations. For simplicity, we assume that the star formation timescale differs between two populations as included in $T_{\mathrm{d}}$ and $T_{\mathrm{d}, \text { col }}$ but the resultant SFE is the same for both populations as $\varepsilon_{\mathrm{SFE}}$.

Given a $n_{\mathrm{cl}}$ and $\varepsilon_{\mathrm{SFE}}$, one can calculate cumulative SFR with this equation. On one hand, we evaluate $n_{\mathrm{cl}}$ directly from the calculated GMCMF. On the other hand, we need to model $\varepsilon_{\mathrm{SFE}}$. In principle, individual GMCs can have different SFE. Observationally, SFE averaged over a galactic disk is equal to a few per cent (Zuckerman \& Evans 1974). In this study, we employ a fixed efficiency 1 per cent as an ensemble-averaged SFE for simplicity. This 1 per cent can be obtained as follows. Given the Salpeter IMF, one massive star $\gtrsim 20 M_{\odot}$ can be born out of $1000 M_{\odot}$ star cluster. Such single massive star may disperse its parental cloud up to $10^{5} M_{\odot}$ according to a detail line-radiation magnetohydrodynamics simulations (Hosokawa \& Inutsuka 2006; Inutsuka et al. 2015). This suggests that SFE is 1 per cent on average $\left(1000 M_{\odot}\right.$ star out of $10^{5} M_{\odot}$ GMC). This efficiency is essentially constant with GMC mass because massive GMCs $>10^{5} M_{\odot}$ create more massive stars and more dispersal. Therefore, we employ $\varepsilon_{\mathrm{SFE}}=0.01$ as our fiducial value.

Note that, this mass-independent SFE is not applicable to the low-mass GMCs $\lesssim 10^{5} M_{\odot}$ because their molecular gas mass is insufficient to produce a massive star that can blow out the entire parental GMC unless the stellar IMF in such low-mass GMCs prefer top-heavy IMF than Salpeter IMF. We are planning to investigate this effect and report in our forthcoming article. Also note that we use $T_{\mathrm{d}}$ and $T_{\mathrm{d} \text {,col }}$ but neither $T_{*}$ nor $T_{\text {dest }}$ in equation (12) because of our definition of SFE.

Figure 4 shows the time evolution of cumulative SFR as a function of GMC mass in solid lines. This shows that the cumulative SFR becomes $\mathcal{O}\left(10^{5}\right) M_{\odot} \mathrm{kpc}^{-3} \mathrm{Myr}^{-1}$, which corresponds to the typical SFR of a few solar mass per year over a galactic disk (e.g., the Milky Way galaxy by Spitzer data Robitaille \& Whitney (2010)). In figure 4, we also plot the CCC-driven cumulative SFR in dotted lines, which is a fraction of total cumulative SFR. This suggests that most of the CCC- 


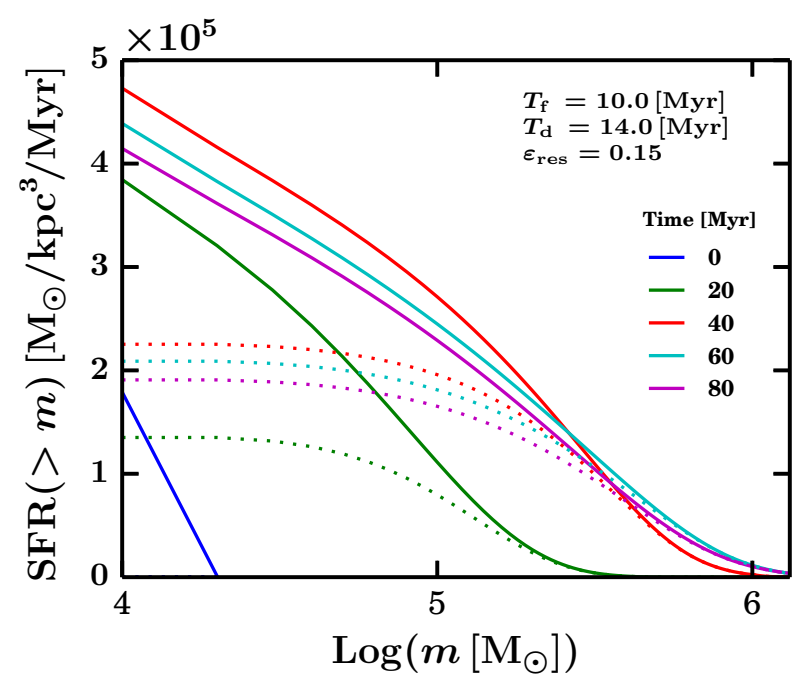

Fig. 4. Cumulative star formation rate $\operatorname{SFR}(>m)$ as a function of GMC mass. The color corresponds to time evolution. The solid lines represent the overall cumulative SFR originating from normal and CCC GMCs, whereas the dotted lines show the cumulative SFR originated only in CCC GMCs. The vertical axis is in the unit of $M_{\odot} \mathrm{kpc}^{-3} \mathrm{Myr}^{-1}$, thus total SFR on a galactic disk whose volume is similar to that of the Milky Way galaxy (e.g., $10 \mathrm{kpc} \times 10 \mathrm{kpc} \times 100 \mathrm{pc}$ ) is a few solar mass per year in the range plotted here. This is a good agreement with observed typical SFR in the Milky Way and nearby galaxies.

driven SFR comes from GMCs with mass $\gtrsim 10^{5.5} M_{\odot}$, where the GMCMF slope is significantly deviated from the steady state power-law slope. In addition, the $\operatorname{SFR}\left(>10^{4} M_{\odot}\right)$ indicates that the CCC-driven SFR may amount to a few 10 per cent (at most half) of the total SFR on galactic disk. Our calculated CCC-driven SFR is presumably overestimated and may correspond to an upper limit because our formulation allows all colliding GMCs to coagulate together even when only their peripheries touch each other. This overestimation is also due to assumed star formation efficiency in CCC GMCs (see subsection 5.2).

As star formation goes, the GMC mass gradually accumulate into stars. Our time-evolution equation (equation (8)) does not explicitly track such mass transformation. Although this is a very gradual process compared with other processes (massgrowth, dispersal, and CCC), the such mass becomes $\sim 10^{8} M_{\odot}$ accumulated over an entire galactic disk, if we integrate the evolution equation more than $100 \mathrm{Myr}$ with a given SFR about a few solar mass per year. This may amount to at least a few per cent of the total molecular gas budget in a single galaxy. Therefore, to extend the current semi-analytical formulation to galaxy evolution over cosmological timescale, mass transformation into stars needs to be formulated. We reserve this longterm evolution for future work. In this case, we also must take into account the gas inflow from halos down to galactic disks, which needs to be conducted together with cosmological largescale structures.

\subsection{Cloud-Cloud Collision Frequency as a Function of GMC Mass}

In this subsection, we quantify the $\mathrm{CCC}$ frequency as a function of GMC mass. We can define two different timescales that characterize CCC process: "number collision timescale" and "mass collision timescale".

Based on the collision term in equation (8), the total number of collisions that a single GMC with mass $m$ experiences per unit time is given as the following integration:

$$
\begin{aligned}
& \int K\left(m, m_{2}\right) n_{\mathrm{cl}, 2} \mathrm{~d} m_{2} \\
= & \int K\left(m, m_{2}\right) n_{\mathrm{cl}, 2} m_{2} \mathrm{~d} \ln m_{2} .
\end{aligned}
$$

Therefore, the CCC event rate between a single GMC with mass $m$ and GMCs with $m_{2}$ with a given differential number density $n_{\mathrm{cl}, 2}$ per unit logarithmic mass interval $\Delta \ln m_{2}$ is

$K\left(m, m_{2}\right) n_{\mathrm{cl}, 2} m_{2}$.

One can evaluate the typical collision timescale for a single GMC with mass $m$ colliding with a GMC with mass $m_{2}$ as

$T_{\mathrm{col}, \operatorname{num}}\left(m, m_{2}\right)=\frac{1}{K\left(m, m_{2}\right) n_{\mathrm{cl}, 2} m_{2}}$.

Let us call this timescale $T_{\text {col,num }}$ as "number collision timescale" because this is an e-folding timescale for the number of GMCs with mass $m$. Similarly, the total mass-gain (i.e., mass-growth) of a single GMC with mass $m$ due to $\mathrm{CCC}$ is given as the following integration:

$$
\begin{aligned}
& \int K\left(m, m_{2}\right) n_{\mathrm{cl}, 2} m_{2} \mathrm{~d} m_{2} \\
= & \int K\left(m, m_{2}\right) n_{\mathrm{cl}, 2} m_{2} m_{2} \mathrm{~d} \ln m_{2} .
\end{aligned}
$$

Therefore, we can also define another typical timescale, over which a GMC with mass $m$ grows in mass due to CCC with GMCs of mass $m_{2}$ per unit logarithmic mass interval $\Delta \ln m_{2}$ as:

$T_{\mathrm{col}, \mathrm{mass}}\left(m, m_{2}\right)=\frac{m}{K\left(m, m_{2}\right) n_{\mathrm{cl}, 2} m_{2} m_{2}}$.

Let us name this timescale $T_{\text {col,mass }}$ as "mass collision timescale" because this is an e-folding timescale for the total mass of GMCs with mass $m$. The CCC frequency for a given GMC population with mass $m$ is therefore characterized as a function of $m_{2}$ by $T_{\mathrm{col}, \mathrm{num}}\left(m, m_{2}\right)$ and $T_{\mathrm{col} \text {,mass }}\left(m, m_{2}\right)$.

Note that our formulation treats the CCC between GMCs with mass $m$ and $m_{2}$ as a coagulation resulting into a GMC with mass $m+m_{2}$. Therefore, $T_{\mathrm{col} \text {,mass }}\left(m, m_{2}\right)$ in the regime of $m \gg m_{2}$ represents the mass-growth timescale of GMCs with mass $m$, which corresponds to the time evolution of GMCMF around mass $m$. However in the regime of $m \ll m_{2}$, the collisional outcome with mass $m+m_{2}$ is significantly larger than 

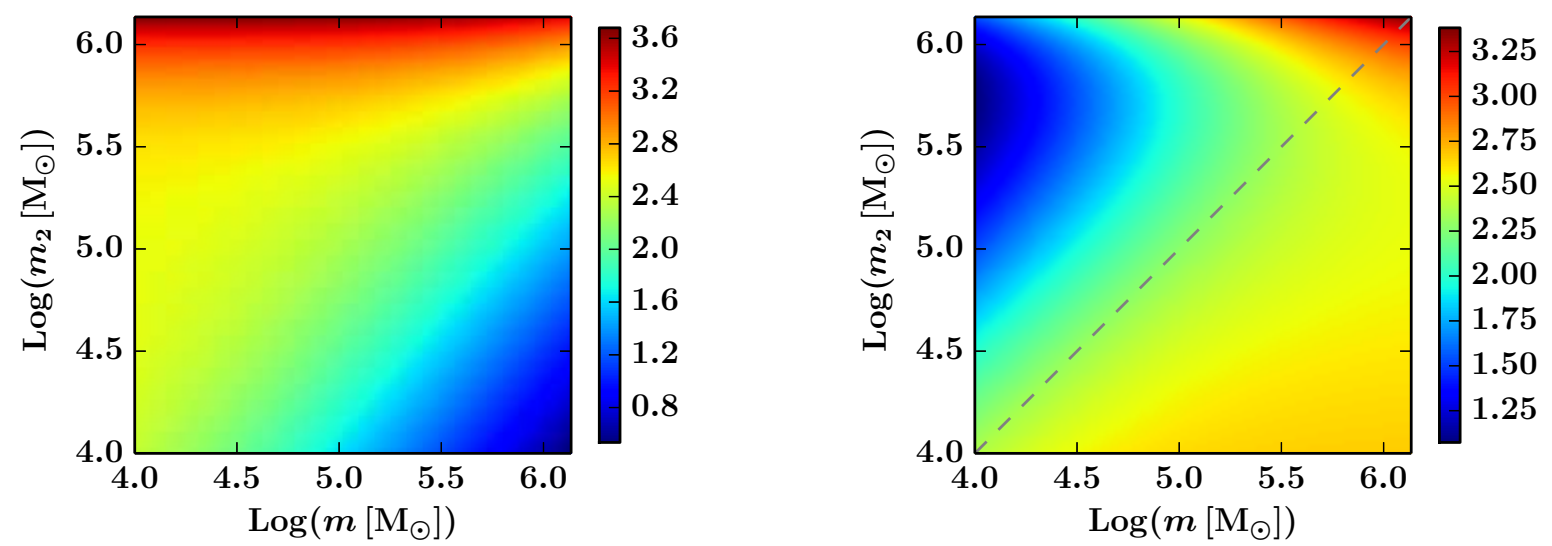

Fig. 5. Left: The typical "number collision timescale" $T_{\text {col, num }}\left(m, m_{2}\right)$ as a function of GMC mass combination involved in a CCC at 60 Myr. The horizontal axis corresponds to $m$ and the vertical axis corresponds to $m_{2}$. The color scale corresponds to $\log _{10}\left(T_{\mathrm{col}, \text { num }}[\mathrm{Myr}]\right)$. Right: The typical "mass collision timescale" $T_{\text {col, mass }}\left(m, m_{2}\right)$ as a function of GMC mass combination involved in a CCC at 60 Myr. The horizontal axis corresponds to $m$ and the vertical axis corresponds to $m_{2}$. The color scale corresponds to $\log _{10}\left(T_{\mathrm{col}, \operatorname{mass}}[\mathrm{Myr}]\right)$. The thin gray dashed line divides the panel into two regimes: $m>m_{2}$ (lower-right) and $m<m_{2}$ (upper-left).

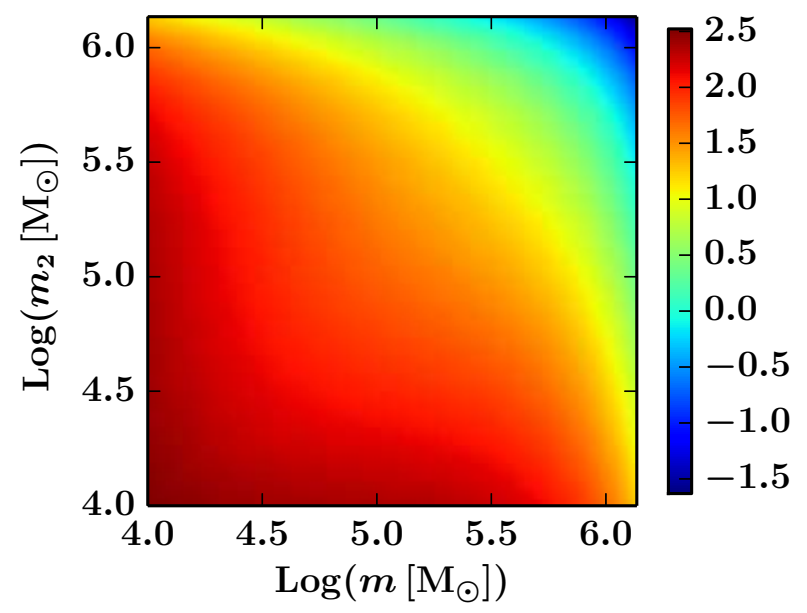

Fig. 6. The expected number of CCC events observed in a galactic disk as a function of GMC mass pairs (i.e., $N_{\mathrm{obs}}$ defined by equation (26)). Here we opt to employ $\Delta T_{\text {obs }}=1 \mathrm{Myr}$ and $V_{\text {survey }}=10 \mathrm{kpc}^{3}$ by assuming an ideal condition where we can observe all the GMCs across the entire galactic disk. The color scale is in the $\log _{10}$ scale. This figure indicates that most of the observationally accessible CCC events occur between GMCs with mass $\lesssim 10^{5.5} M_{\odot}$, whereas the CCC between massive GMCs, which presumably gives significant impact on CCC-driven star formation, may not be observed.

a GMC with mass $m$, thus $T_{\text {col,mass }}\left(m, m_{2}\right)$ does not necessarily represent the time evolution of GMCMFs. For example, a CCC between GMCs with mass $10^{4} M_{\odot}$ and $10^{6} M_{\odot}$ forms a GMC with mass $1.01 \times 10^{6} M_{\odot}$. For the GMC with mass $10^{4} M_{\odot}$, this coagulation effectively looks like rapid massgrowth. However, in terms of the GMCMF time evolution, this appears as the gradual mass-growth of GMCs at mass $10^{6} M_{\odot}$. It is thus more useful to compare $T_{\mathrm{col} \text {,mass }}\left(m, m_{2}\right)$ only in the regime of $m \geq m_{2}$ with other timescales (e.g., $T_{\mathrm{f}}$ and $\left.T_{\mathrm{d}}\right)$ when we discuss the time evolution of GMCMFs.

\subsubsection{Number Collision Timescale}

The left panel in figure 5 shows $T_{\text {col,num }}$ as a function of mass pair in one CCC event. Based on its definition in equation (15), $T_{\text {col,num }}\left(m, m_{2}\right)$ represents the e-folding timescale for a single GMC with mass $m$ due to the collisions with GMCs with mass $m_{2}$. Thus the physical meaning of $T_{\text {col,num }}\left(m, m_{2}\right)$ is different from that of $T_{\text {col,num }}\left(m_{2}, m\right)$. Indeed, figure 5 shows such asymmetry between $m$ and $m_{2}$. Note that, $T_{\text {col,num }}\left(m, m_{2}\right)$ and $T_{\text {col,num }}\left(m_{2}, m\right)$ differ from the total collisional event rate between $m$ and $m_{2}$, which is symmetric between $m$ and $m_{2}$. We discuss the total collisional event rate in section 4.3.3.

The figure indicates that massive GMCs (i.e., larger mass range in the horizontal axis) have higher opportunity to collide with smaller clouds than with massive clouds because the number density of smaller clouds is larger than that of massive clouds. Due to the same reason, smaller GMCs (i.e., smaller mass range in the horizontal axis) also have higher opportunity to collide with smaller clouds than with massive clouds. Such intuitive understanding can be analytically confirmed by equation (15) as follows. In $m \gg m_{2}$ regime, $T_{\text {col, num }}$ can be written as:

$T_{\mathrm{col}, \mathrm{num}}\left(m, m_{2}\right) \propto \frac{1}{m m_{2}^{1-\alpha}}$.

Therefore $T_{\text {col,num }}$ becomes longer with $m_{2}$ as $T_{\text {col,num }} \propto m_{2}^{0.7}$ given the typical GMCMF slope $-\alpha \sim-1.7$ as shown in figure 3. This corresponds to the increasing trend of $T_{\text {col,num }}$ along the vertical axis at a given large mass in the horizontal mass coordinate in figure 5 . Note that $T_{\mathrm{col}, \mathrm{num}}\left(m, m_{2}\right)$ increases faster than $m_{2}^{0.7}$ in the range of $m_{2}>10^{5.5} M_{\odot}$. In this regime, the GMCMF deviates from the power-law distribution assumed in equation 18 . Thus, $T_{\mathrm{col}, \text { num }}\left(m, m_{2}\right)$ rapidly increases as $n_{\mathrm{cl}, 2}$ decreases with $m_{2}$. The resultant difference in $T_{\mathrm{col}, \text { num }}$ is two or more orders of magnitude (e.g., between 
$T_{\text {col,num }}\left(10^{6} M_{\odot}, 10^{4} M_{\odot}\right)$ and $\left.T_{\mathrm{col}, \mathrm{num}}\left(10^{6} M_{\odot}, 10^{6} M_{\odot}\right)\right)$. On the other hand, in $m \lesssim m_{2}$ regime, $T_{\mathrm{col}, \mathrm{num}}$ can be evaluated as:

$T_{\text {col,num }}\left(m, m_{2}\right) \propto \frac{1}{m_{2}^{2-\alpha}}$.

Therefore $T_{\text {col,num }}$ becomes shorter with $m_{2}$ as $T_{\text {col,num }} \propto$ $m_{2}^{-0.3}$ given the typical GMCMF slope $-\alpha \sim-1.7$ as shown in figure 3 . This corresponds to the decreasing trend of $T_{\text {col,num }}$ along the vertical axis at a given small mass in the horizontal mass coordinate in figure 5. However, its dependence on $m_{2}$ is limited to the power of $2-\alpha=0.3$ thus this trend is difficult to recognize in figure 3. In addition, similar to equation (18), the power-law GMCMF assumption in equation (19) is invalid in the range of $m_{2}>10^{5.5} M_{\odot}$. Thus $T_{\text {col,num }}\left(m, m_{2}\right)$ increases rapidly as $n_{\mathrm{cl}, 2}$ decreases with $m_{2}$.

Note that the above discussion is based on GMCMF with $-\alpha \sim-1.7$ assuming that this represents overall averaged GMC population on a galactic disk. However, in case of inter-arm regions with $-\alpha<-2$ for example, $T_{\text {col,num }}$ is always increasing function with $m_{2}$ because the number of large clouds is very few.

\subsubsection{Mass Collision Timescale}

As seen in section 4.3.1, $T_{\mathrm{col} \text {,num }}$ characterizes the frequency of individual CCC events that a single GMC with mass $m$ experiences. However this does not always characterize impact on GMCMF evolution, because, for example, collision with small clouds may not largely contribute to mass-growth of massive clouds, which is not appreciable in GMCMF evolution. Therefore, we evaluate the mass-growth driven by CCC by calculating $T_{\text {col,mass }}$, which gives the typical e-folding time in mass for a single GMC with mass $m$ by CCC. The right panel in figure 5 shows $T_{\text {col,mass }}$ as a function of GMC masses in a given GMC pair. This panel indicates that the mass-growth of massive clouds is still dominated by CCC with small to intermediate mass clouds. However, such CCC events increase only limited amount of mass so that $T_{\mathrm{col} \text {,mass }}$ has only up to one order of magnitude difference from that of CCCs between massive GMCs.

These trends can be analytically confirmed by equation (17) as follows, similar to the discussion for $T_{\text {col,num. In }} m \gg m_{2}$ regime, $T_{\mathrm{col} \text {, mass }}$ can be written as:

$T_{\text {col,mass }}\left(m, m_{2}\right) \propto \frac{1}{m_{2}^{2-\alpha}}$.

Therefore $T_{\text {col,mass }}$ becomes shorter with $m_{2}$ as $T_{\text {col,mass }} \propto$ $m_{2}^{-0.3}$ given the typical GMCMF slope $-\alpha \sim-1.7$ as shown in figure 3 . This corresponds to the decreasing trend of $T_{\text {col,mass }}$ along the vertical axis at a given large mass in the horizontal mass coordinate in figure 5. Again, the rapid increment in $T_{\text {col,mass }}$ in the range of $m_{2}>10^{5.5} M_{\odot}$ corresponds to the deviation of the GMCMF from the power-law distribution in this mass range.
Similarly, in $m \lesssim m_{2}$ regime, $T_{\text {col,mass }}$ can be evaluated as: $T_{\text {col,mass }}\left(m, m_{2}\right) \propto \frac{m}{m_{2}^{3-\alpha}}$.

Therefore $T_{\text {col,mass }}$ becomes shorter with $m_{2}$ as $T_{\text {col,mass }} \propto$ $m_{2}^{-1.3}$ given the typical GMCMF slope $-\alpha \sim-1.7$ as shown in figure 3 . This corresponds to the decreasing trend of $T_{\text {col,mass }}$ along the vertical axis at a given small mass in the horizontal mass coordinate in figure 5 . The slight increment in $T_{\text {col,mass }}$ in the range of $m_{2}>10^{5.5} M_{\odot}$ corresponds to the deviation of the GMCMF from the power-law distribution in this mass range.

Note that, as we have already discussed in the paragraph following equation (17), only the right-lower half of this panel (i.e., $m \geq m_{2}$ regime) can be directly compared with other timescales governing the GMCMF evolution (e.g., $T_{\mathrm{f}}$ and $T_{\mathrm{d}}$ ). In this regime, the typical $T_{\mathrm{col} \text {,mass }}$ has the order of $100 \mathrm{Myr}$, which is still longer than $T_{\mathrm{d}}$ or $T_{\mathrm{d} \text {,col}}$. Therefore, the massive-end of GMCMF does not show significant growth after $60 \mathrm{Myr}$.

\subsubsection{Observability}

In this sub-subsection, we explore simple estimation of $\mathrm{CCC}$ observability in galactic disks. The observability of CCC between GMCs with mass $m$ and $m_{2}, f_{\text {obs }}\left(m, m_{2}\right)$, can be characterized by multiplying its frequency and number density of GMCs:

$f_{\text {obs }}\left(m, m_{2}\right)=\frac{\Delta n(m)}{T_{\text {col, num }}\left(m, m_{2}\right)}$.

The number density $\Delta n(m)$ can be estimated from $n_{\text {cl }}$ calculated in our GMCMF time evolution as:

$$
\begin{aligned}
\Delta n(m) & =\int_{m}^{m+\Delta m} n_{\mathrm{cl}} \mathrm{d} m \\
& =\int_{\ln m}^{\ln (m+\Delta m)} n_{\mathrm{cl}} m \mathrm{~d} \ln m .
\end{aligned}
$$

This integration width $\Delta m$ is in principle determined by the capability of individual observations. Instead of specifying any capability, we here employ the number density per unit logarithmic mass interval $\Delta \ln m=\ln (m+\Delta m)-\ln m=1$ for simplicity, thus

$\Delta n(m)=n_{\mathrm{cl}} m$

This choice of $\Delta n(m)$ is consistent with the fact that we employ unit logarithmic mass interval $\Delta \ln m$ in equation (17) to define $T_{\mathrm{col}, \mathrm{num}}$. This definition makes the observability $f_{\mathrm{obs}}\left(m, m_{2}\right)$ symmetric between $m$ and $m_{2}$ as:

$f_{\mathrm{obs}}\left(m, m_{2}\right)=K\left(m, m_{2}\right) n_{\mathrm{cl}, 2} m_{2} n_{\mathrm{cl}} m$.

Such symmetry must exist because, unlike $T_{\mathrm{col}, \text { num }}$, the number of total collisional events itself cannot distinguish between $m$ and $m_{2}$.

One can estimate the number of events expected to be observed within a galactic disk by multiplying $f_{\text {obs }}$, the duration over which observation can identify colliding GMCs as a CCC 
event, $\Delta T_{\mathrm{CCC}}$, and the survey volume $V_{\text {survey }}$ :

$N_{\text {obs }}\left(m, m_{2}\right)=f_{\text {obs }}\left(m, m_{2}\right) \Delta T_{\mathrm{CCC}} V_{\text {survey }}$

As a demonstration, we make a simple prediction for future surveys under an ideal condition that we resolve and identify all the GMC populations across an entire galactic disk. Figure 6 shows the resultant $N_{\text {obs }}$ where we assume $\Delta T_{\mathrm{CCC}} \sim 1 \mathrm{Myr}$ and $V_{\text {survey }} \sim 10 \mathrm{kpc}^{3}$, which corresponds to the total volume of Milky Way galactic thin disk in which GMCs most likely reside. Our result suggests that we may observe over 100 events of CCC between $10^{4} M_{\odot}$. Indeed, most of the observed CCC candidates to date involve $\mathcal{O}\left(10^{4}\right) M_{\odot}$ GMCs (e.g., Fukui et al. 2016). On the other hand, our result also indicates that it is less likely to observe the CCC events between GMCs with mass $>10^{5.5} M_{\odot}$ that play a dominant role in CCC-driven SFR.

\section{DISCUSSION}

\subsection{Correction Factor in Cloud-Cloud Collisions}

As shown in equation (4) in section 2.3, there is a correction factor $c_{\mathrm{col}}$ to evaluate the $\mathrm{CCC}$ rate. There, we already mentioned two effects that are considered in this correction factor: gravitational focusing effect and angle with which GMCs collide each other. In this subsection, we briefly introduce and explore several other factors about which the present authors are frequently asked. In the calculations we show in previous sections, we assume that these factors cancel out each other for simplicity, because each factor either increase or decrease the CCC rate by a factor few.

Column Density of GMCs: Latest observations suggest that GMC mean column density could have its peak at $(2-$ 6) $\times 10^{21} \mathrm{~cm}^{-2}$ (e.g., Auriga-California: Harvey et al. (2013), Cygnus X: Schneider et al. (2016), etc.). This corresponds to visual extinction a few, which is able to protect $\mathrm{CO}$ molecules that radio surveys observe. Our fiducial column density is $\Sigma_{\text {mol }}=10^{22} \mathrm{~cm}^{-2}$, a factor few denser than the density suggested by the latest observations. Therefore, the assumed CCC rate may be biased lower by a factor few.

Number Density and Relative Velocity: Observationally, both the number density and cloud-to-cloud velocity dispersion of GMCs increase towards galactic centers (e.g., Central Molecular Zone in the case of the Milky Way galaxy: Morris \& Serabyn (1996)). Therefore, the CCC rate is also enhanced at galactic centers. Investigation in further inner region of galactic disks needs to take into account such variation. However in this study, we restrict ourselves to a disk region, for example the solar circle in the case of the Milky Way galaxy. Also, super star cluster formation sites show high relative velocities 10 - $20 \mathrm{~km} \mathrm{~s}^{-1}$ (e.g., Furukawa et al. 2009; Ohama et al. 2010; Fukui et al. 2014, 2016), where super star cluster is defined as star clusters having $10-20 \mathrm{O}$ stars. Such possibility that mas- sive star formation is enhanced as a function of relative velocity between GMCs also has to be investigated, but we focus on a simple question how much star formation can be induced by $\mathrm{CCC}$ at a given star formation efficiency in this study. Note that, at galactic centers, the magnetic field strength was reported to be very large (e.g., a few milli-Gauss), at least, locally. This stronger fields presumably modifies self-growth timescale $T_{\mathrm{f}}$ as well. Note also that, in non-disk small galaxies or some specific volume in galactic disks, molecular cloud formation and subsequent star cluster formation can be triggered by large-scale colliding Hi flow (e.g., Fukui et al. 2017b, : inflow from Small Magellanic Cloud onto Large Magellanic Cloud), which needs to be investigated.

Area for One-Zone: Our time-evolution equation is essentially one-zone and calculates the differential number density of GMC populations $n_{\mathrm{cl}}$, which therefore does not require any specified three-dimensional configuration for concerned volume in which calculated GMCMFs exist under CCC-absent cases. Nevertheless, one can still estimate calculated volume that is self-consistent within our modeling. For example, GMCs can travel roughly $1 \mathrm{kpc}$ in $100 \mathrm{Myr}$ with a proper velocity of $10 \mathrm{kms}^{-1}$. Therefore, our calculated GMCMF up to $100 \mathrm{Myr}$ should correspond to ensemble population of GMCs in a cylinder with a surface area of $1 \mathrm{kpc}^{2}$ and with a depth of $100 \mathrm{pc}$ given that a galactic thin disk has a scale height $100 \mathrm{pc}$ in which GMCs populates (see section 2). It is less likely to have GMC collisions beyond this cylinder. However, to enable comparisons with observations, we assume that the area is bigger than $1 \mathrm{kpc}^{2}$ by referring to the observed area covered by subdivided regions (e.g., PAWS Colombo et al. (2014a): from $7.54 \mathrm{kpc}^{2}$ in arm regions to $19.99 \mathrm{kpc}^{2}$ in inter-arm regions), because GMCs are statistically able to collide each other even if they are apart more than $1 \mathrm{kpc}$ in each subdivided area. In our calculation, this overestimated area overestimate the cumulative number of massive-end GMCs so that we invoke collisions with GMCs whose cumulative number is less than 1 . In this article, we aim at demonstrating our modeling for a typical region in a galactic disk so that we opt to employ a cylinder with $10 \mathrm{kpc}^{2}$ area and with 100 pc depth.

\subsection{Overestimation in Triggered Star Formation}

As described in subsection 4.2, we assume that SFE in CCC sites is 1 per cent $\left(\varepsilon_{\mathrm{SFE}}=0.01\right)$ of the coagulated parental GMC mass. However, SFE could be simply limited by 1 per cent of the smaller GMC in a CCC pair. In case of a GMC pair with large mass difference, masses $10^{4} M_{\odot}$ and $10^{6} M_{\odot}$ for example, the resultant star cluster mass can be $\sim 10^{2} M_{\odot}=1 \%$ of $10^{4} M_{\odot}$, whereas our calculation estimates this as $\sim 10^{4} M_{\odot}=$ $1 \%$ of $10^{6} M_{\odot}$. Therefore, in a CCC pair with large mass difference, our SFE becomes close to 100 per cent out of smaller 
GMCs.

Radio observations of CCC sites (e.g., Fukui et al. 2016) suggest that at least 1 per cent of the smaller GMC mass in a given GMC pair turns into massive stars $>20 M_{\odot}$ and they could form as many massive stars equally as low-mass stars. Detailed magnetohydrodynamics simulations (c.f. Inoue et al. 2017) indicates that molecular cloud cores may have a flat IMF, although with poor statistics due to small number of samples. Only if stellar population follows Salpeter IMF even in CCC sites, then the number of simultaneously-formed low-mass stars $<20 M_{\odot}$ is larger than that of massive stars thus the total stellar mass can be comparable to colliding GMC mass, i.e., up to 100 per cent SFE of colliding GMC mass.

Therefore, if actual CCC sites preferentially form massive stars our calculated SFR triggered by CCC could be overestimated by a factor from a few to ten. This overestimation impacts the total SFR shown in figure 4 because CCC takes place most frequently between massive GMCs and small GMCs (see figure 5).

Coupled with possible overestimation in the $\mathrm{CCC}$ rate due to the perfect-inelastic assumption (see subsection 3.3), we interpret our calculated SFR driven by CCC as an upper limit.

\subsection{Lifetime and Age of GMCs}

The lifetime and age of GMCs are another two important quantities to understand star formation and star cluster formation along with galaxy evolution (e.g., multiplicity of stellar ages in individual star clusters, migration history of our solar system, and so on). We here distinguish "lifetime" and "age"of GMCs; we define lifetime as the duration time for a GMC to its complete dispersal once star formation starts to take place, whereas age as the overall duration from GMC formation to its complete dispersal.

On one hand, the typical lifetime in our calculation corresponds to $T_{\mathrm{d}}=14 \mathrm{Myr}$. Some observations indicate such short lifetime (20-30 Myr) inferred from GMC classification in Large Magellanic Cloud (Kawamura et al. 2009) and an upper limit (30 Myr) estimated by GMC number counts in inter-arm regions in Galaxy M51 (Meidt et al. 2015). On the other hand, we have a delta function like mass distribution as the initial condition of our calculation where the minimum-mass GMCs alone exist. This enables us to highlight how fast GMCs can grow. To grow in mass, GMCs have to survive stellar feedback whose rate is determined by $T_{\mathrm{d}}$ (i.e., more massive GMCs have older ages). Therefore, the age is in general longer than the lifetime. Figure 3 suggests that the GMC age is $\gtrsim 40(80) \mathrm{Myr}$, which GMCs require to grow from the minimum-mass $10^{4} M_{\odot}$ to $\gtrsim 10^{6}\left(10^{7}\right) M_{\odot}$. Such longevity is indicated by observations within the Milky Way galaxy (e.g., Barnes et al. 2011, 2016 and Barnes et al 2017, ApJ, submitted; c.f., Kauffmann et al. 2013) and in nearby galaxies (e.g., Koda et al. 2009).

By this analysis, we presume that observed short lifetimes are likely "lifetime" determined by GMC dispersal rate, whereas observed long lifetimes mostly correspond to "age" rather than lifetime.

\subsection{Background}

In our current calculation, we assume that the background reservoir is always plenty enough to sustain the steady state of GMCMF. However, it is known in the Milky Way and nearby galaxies that the gas distribution (especially atom-to-molecular ratio) varies with the galactocentric radii (c.f., Nakanishi \& Sofue 2016). Therefore, it is desired to investigate the background gas evolution coupled with galactic environment, which we reserve for future works.

\subsection{CCC-driven Star Formation}

In the present article, we calculate and show CCC-driven SFR with a set of typical galactic disk parameters. Our next subject is to compare CCC-driven SFRs between arm and inter-arm regions. Intuitively, high CCC-driven SFR is expected in arm regions because the mass budget in arm regions is dominated by massive GMCs. However, mass-growth by multiple episodes of compression is also fast in arm regions (i.e., short $T_{\mathrm{f}}$ ) to quickly create large amount of normal GMC populations. Thus, it is not obvious whether or not the "fraction" of CCC-driven SFR out of total SFR is high in arm regions, and vice versa for inter-arm regions. Indeed, our pilot calculations indicate that CCC-driven SFR covers 30-50 per cent of star formation in arm regions and $20-40$ per cent in inter-arm regions. Time evolution of GMCMF and subsequent CCC-driven SFR have to be investigated further along with the migration of GMC groups between different regions (e.g., arm to inter-arm and back into arm). This involves time evolution in parameters (especially $T_{\mathrm{f}}, T_{\mathrm{d}}, \varepsilon_{\mathrm{res}}$ ) and is left for future studies.

\section{SUMMARY}

We have performed integration of time evolution equation for giant molecular cloud (GMC) mass functions including cloudcloud collisions and subsequent star formation due to the collisions. Our results indicate that the stellar feedback triggered by cloud-cloud collisions modify only the massive end of GMC mass functions. Thus the mass functions exhibit power-law slopes in the low-mass regime $\left(\lesssim 10^{5.5} M_{\odot}\right)$, which can be characterized as a ratio of the GMC formation timescale to their dispersal timescale according to the environment in galactic disks. The star formation rate (SFR) calculated with a given GMC mass function indicates that a few 10 per cent (at most half) of the galactic star formation may be operated by cloud-cloud 
collisions (CCCs). This analysis is based on assumptions where 1) GMC population that experience cloud-cloud collisions have a shorter star formation timescale than normal GMC population without cloud-cloud collisions, and 2) both populations have the same star formation efficiency of 1 per cent with the Salpeter initial mass function. Cloud-cloud collisions may play a more important role if they would result in higher star formation efficiency. Lastly, we also indicate that CCC between smaller clouds $\left(\sim 10^{4} M_{\odot}\right)$ are more probable to be observed due to its large number, although most of the CCC-driven SFR is triggered by CCC between massive GMCs $\gtrsim 10^{5.5} M_{\odot}$.

\section{ACKNOWLEDGEMENT}

We are grateful to the anonymous referee and our editor Asao Habe for carefully reading our manuscript and providing thoughtful comments, which improved our manuscript in great details. MINK (15J04974), HK (26287101, 17K05632, 17H01105, 17H01103), SI (23244027, 16H02160), and YF (15H05694) are supported by Grants-in-Aid from the Ministry of Education, Culture, Sports, Science, and Technology of Japan. MINK thank Kengo Tachihara, Hidetoshi Sano, Atsushi Nishimura, Dario Colombo, Veselina Kalinova, Jens Kauffmann, Thushara Pillai, Karl Menten, Eva Schinnerer, Sharon Meidt, Henrik Beuther, Juan Soler, Jouni Kainulainen, Dimitrios Gouliermis, Maria Jesus Jimenez, Takuma Kokusho, and Doris Arzoumanian for educating us with observational backgrounds. MINK also appreciate Tsuyoshi Inoue, Hosokawa Takashi, Kazunari Iwasaki, Kengo Tomida, Kentaro Nagamine, Jonathan Tan, Stefanie Walch, Daniel Seifried, Diederik Kruijssen, Melanie Chevance, Sarah Jeffreson, Daniel Haydon, Marta Reina-Campos, Ralf Klessen, Eric Pellegrini, Daniel Rahner, and Bhaskar Agarwal for fruitful discussion, and Keiichi Kodaira, Hiroshi Karoji, Philippe Andre, Patrick Hennebelle, and Enrique Vazquez-Semadeni for encouraging MINK throughout this project.

\section{References}

André, P., Men'shchikov, A., Könyves, V., \& Arzoumanian, D. 2011, in IAU Symposium, Vol. 270, Computational Star Formation, ed J. Alves, B. G. Elmegreen, J. M. Girart, \& V. Trimble, 255-262

André, P., Men'shchikov, A., Bontemps, S., et al. 2010, A\&A, 518, L102 Audit, E., \& Hennebelle, P. 2005, A\&A, 433, 1

Audit, E., \& Hennebelle, P. 2008, in Astronomical Society of the Pacific Conference Series, Vol. 385, Numerical Modeling of Space Plasma Flows, ed. N. V. Pogorelov, E. Audit, \& G. P. Zank, 73

Barnes, P. J., Hernandez, A. K., O’Dougherty, S. N., Schap, III, W. J., \& Muller, E. 2016, ApJ, 831, 67

Barnes, P. J., Yonekura, Y., Fukui, Y., et al. 2011, ApJS, 196, 12

Bronfman, L., Casassus, S., May, J., \& Nyman, L.-Å. 2000, A\&A, 358, 521

Colombo, D., Hughes, A., Schinnerer, E., et al. 2014a, ApJ, 784, 3
Colombo, D., Meidt, S. E., Schinnerer, E., et al. 2014b, ApJ, 784, 4

Cowie, L. L. 1980, ApJ, 236, 868

Dame, T. M., Ungerechts, H., Cohen, R. S., et al. 1987, ApJ, 322, 706

Dewangan, L. K. 2017, ApJ, 837, 44

Dewangan, L. K., Ojha, D. K., Luna, A., et al. 2016, ApJ, 819, 66

Dobashi, K., Matsumoto, T., Shimoikura, T., et al. 2014, ApJ, 797, 58

Dobbs, C. L., \& Pringle, J. E. 2013, MNRAS, 432, 653

Dobbs, C. L., Pringle, J. E., \& Duarte-Cabral, A. 2015, MNRAS, 446 , 3608

Engargiola, G., Plambeck, R. L., Rosolowsky, E., \& Blitz, L. 2003, ApJS, 149,343

Field, G. B., Goldsmith, D. W., \& Habing, H. J. 1969, ApJL, 155, L149

Fukui, Y., Hattori, Y., Torii, K., et al. 2017a, ArXiv e-prints, arXiv:1706.08720

Fukui, Y., Tsuge, K., Sano, H., et al. 2017b, PASJ, 69, L5

Fukui, Y., Ohama, A., Hanaoka, N., et al. 2014, ApJ, 780, 36

Fukui, Y., Harada, R., Tokuda, K., et al. 2015, ApJL, 807, L4

Fukui, Y., Torii, K., Ohama, A., et al. 2016, ApJ, 820, 26

Fukui, Y., Torii, K., Hattori, Y., et al. 2017c, ArXiv e-prints, arXiv:1701.04669

Fukui, Y., Kohno, M., Yokoyama, K., et al. 2017d, ArXiv e-prints, arXiv:1706.05768

—. 2017e, ArXiv e-prints, arXiv:1706.05771

Furukawa, N., Dawson, J. R., Ohama, A., et al. 2009, ApJL, 696, L115

Harayama, Y., Eisenhauer, F., \& Martins, F. 2008, ApJ, 675, 1319

Harvey, P. M., Fallscheer, C., Ginsburg, A., et al. 2013, ApJ, 764, 133

Hayashi, K., Sano, H., Enokiya, R., et al. 2017, ArXiv e-prints, arXiv:1706.05871

Heitsch, F., Burkert, A., Hartmann, L. W., Slyz, A. D., \& Devriendt, J. E. G. 2005, ApJL, 633, L113

Heitsch, F., Slyz, A. D., Devriendt, J. E. G., Hartmann, L. W., \& Burkert, A. 2006, ApJ, 648, 1052

Hennebelle, P., \& Audit, E. 2007, A\&A, 465, 431

Hennebelle, P., Audit, E., \& Miville-Deschênes, M.-A. 2007, A\&A, 465, 445

Hosokawa, T., \& Inutsuka, S.-i. 2006, ApJL, 648, L131

Inoue, T., \& Fukui, Y. 2013, ApJL, 774, L31

Inoue, T., Hennebelle, P., Fukui, Y., et al. 2017, ArXiv e-prints, arXiv:1707.02035

Inoue, T., \& Inutsuka, S.-i. 2008, ApJ, 687, 303

-. 2012, ApJ, 759, 35

Inutsuka, S.-i. 2001, ApJL, 559, L149

Inutsuka, S.-i., Inoue, T., Iwasaki, K., \& Hosokawa, T. 2015, A\&A, 580, A49

Kauffmann, J., Pillai, T., \& Goldsmith, P. F. 2013, ApJ, 779, 185

Kawamura, A., Mizuno, Y., Minamidani, T., et al. 2009, ApJS, 184, 1

Kennicutt, R. C., \& Evans, N. J. 2012, ARA\&A, 50, 531

Kim, J.-G., Kim, W.-T., \& Ostriker, E. C. 2016, ApJ, 819, 137

Kobayashi, M. I. N., Inutsuka, S.-i., Kobayashi, H., \& Hasegawa, K. 2017, ApJ, 836, 175

Koda, J., Scoville, N., Sawada, T., et al. 2009, ApJL, 700, L132

Koda, J., Sawada, T., Wright, M. C. H., et al. 2011, ApJS, 193, 19

Koda, J., Scoville, N., Hasegawa, T., et al. 2012, ApJ, 761, 41

Kohno, M., Torii, K., Tachihara, K., et al. 2017, ArXiv e-prints, arXiv:1706.07964

Koyama, H., \& Inutsuka, S.-i. 2002, ApJL, 564, L97

Kudryavtseva, N., Brandner, W., Gennaro, M., et al. 2012, ApJL, 750, L44 
Kwan, J. 1979, ApJ, 229, 567

Kwan, J., \& Valdes, F. 1983, ApJ, 271, 604

Leroy, A. K., Schinnerer, E., Hughes, A., et al. 2017, ArXiv e-prints, arXiv: 1706.08540

Levinson, F. H., \& Roberts, Jr., W. W. 1981, ApJ, 245, 465

Malyshkin, L., \& Goodman, J. 2001, Icarus, 150, 314

Meidt, S. E., Hughes, A., Dobbs, C. L., et al. 2015, ApJ, 806, 72

Morris, M., \& Serabyn, E. 1996, ARA\&A, 34, 645

Muñoz, D. J., Mardones, D., Garay, G., et al. 2007, ApJ, 668, 906

Nakamura, F., Miura, T., Kitamura, Y., et al. 2012, ApJ, 746, 25

Nakamura, F., Sugitani, K., Tanaka, T., et al. 2014, ApJL, 791, L23

Nakanishi, H., \& Sofue, Y. 2016, PASJ, 68, 5

Nishimura, A., Costes, J., Inaba, T., et al. 2017a, ArXiv e-prints, arXiv: 1706.06002

Nishimura, A., Minamidani, T., Umemoto, T., et al. 2017b, ArXiv eprints, arXiv: 1706.06956

Ohama, A., Dawson, J. R., Furukawa, N., et al. 2010, ApJ, 709, 975

Ohama, A., Tsutsumi, D., Sano, H., et al. 2017a, ArXiv e-prints, arXiv: 1706.05652

Ohama, A., Kono, M., Tsutsumi, D., et al. 2017b, ArXiv e-prints, arXiv:1706.05659

Ohama, A., Kohno, M., Hasegawa, K., et al. 2017c, ArXiv e-prints, arXiv: 1709.02320

Onishi, T., Kawamura, A., Abe, R., et al. 1999, PASJ, 51, 871

Rahner, D., Pellegrini, E. W., Glover, S. C. O., \& Klessen, R. S. 2017, ArXiv e-prints, arXiv: 1704.04240

Robitaille, T. P., \& Whitney, B. A. 2010, ApJL, 710, L11

Rosolowsky, E., Engargiola, G., Plambeck, R., \& Blitz, L. 2003, ApJ, 599, 258

Rosolowsky, E., Keto, E., Matsushita, S., \& Willner, S. P. 2007, ApJ, 661, 830

Roy, A., André, P., Arzoumanian, D., et al. 2015, A\&A, 584, A111

Saigo, K., Onishi, T., Nayak, O., et al. 2017, ApJ, 835, 108

Sano, H., Torii, K., Saeki, S., et al. 2017a, ArXiv e-prints, arXiv: 1708.08149

Sano, H., Enokiya, R., Hayashi, K., et al. 2017b, ArXiv e-prints, arXiv: 1706.05763

Schinnerer, E., Meidt, S. E., Pety, J., et al. 2013, ApJ, 779, 42

Schneider, N., Bontemps, S., Motte, F., et al. 2016, A\&A, 587, A74

Scoville, N. Z., \& Hersh, K. 1979, ApJ, 229, 578

Stark, A. A., \& Brand, J. 1989, ApJ, 339, 763

Stark, A. A., \& Lee, Y. 2005, ApJL, 619, L159

-. 2006, ApJL, 641, L113

Tachihara, K., Mizuno, A., \& Fukui, Y. 2000, ApJ, 528, 817

Takahira, K., Shima, K., Tasker, E. J., \& Habe, A. 2017, ArXiv e-prints, arXiv: 1706.08656

Takahira, K., Tasker, E. J., \& Habe, A. 2014, ApJ, 792, 63

Tasker, E. J., \& Tan, J. C. 2009, ApJ, 700, 358

Tomisaka, K. 1984, PASJ, 36, 457

-. 1986, PASJ, 38, 95

Torii, K., Enokiya, R., Sano, H., et al. 2011, ApJ, 738, 46

Torii, K., Hasegawa, K., Hattori, Y., et al. 2015, ApJ, 806, 7

Torii, K., Hattori, Y., Hasegawa, K., et al. 2017a, ApJ, 840, 111

Torii, K., Hattori, Y., Matsuo, M., et al. 2017b, ArXiv e-prints, arXiv: 1706.07164

Torii, K., Hattori, Y., Hasegawa, K., et al. 2017c, ApJ, 835, 142

Trubnikov, B. A. 1971, Soviet Physics Doklady, 16, 124

Tsuboi, M., Miyazaki, A., \& Uehara, K. 2015, PASJ, 67, 90
Tsutsumi, D., Ohama, A., Okawa, K., et al. 2017, ArXiv e-prints, arXiv: 1706.05664

Vázquez-Semadeni, E., Ryu, D., Passot, T., González, R. F., \& Gazol, A. 2006, ApJ, 643, 245

Walder, R., \& Folini, D. 1998a, A\&A, 330, L21

-. 1998b, Ap\&SS, 260, 215

Williams, J. P., Blitz, L., \& McKee, C. F. 2000, Protostars and Planets IV, 97

Wolfire, M. G., Hollenbach, D., McKee, C. F., Tielens, A. G. G. M., \& Bakes, E. L. O. 1995, ApJ, 443, 152

Wolfire, M. G., McKee, C. F., Hollenbach, D., \& Tielens, A. G. G. M. 2003, ApJ, 587, 278

Wu, B., Tan, J. C., Christie, D., et al. 2017, ApJ, 841, 88

Zuckerman, B., \& Evans, II, N. J. 1974, ApJL, 192, L149 